\title{
Peso à Cobrição e Ganho de Peso Durante a Gestação de Cabras Nativas, Exóticas e Mestiças no Semi-Árido ${ }^{1}$
}

\section{Geovergue Rodrigues de Medeiros ${ }^{2}$, Edgard Cavalcanti Pimenta Filho ${ }^{3}$, Wandrick Hauss de Sousa ${ }^{4}$, Evaneusa Alves de Brito ${ }^{4}$}

\begin{abstract}
RESUMO - Avaliaram-se os efeitos de mês, ano, ordem de parto e tipo de parto sobre o peso à cobrição (PC) e o ganho de peso durante a gestação (GP), respectivamente, de 753 e 527 cabras nativas Canindés, 463 e 333 cabras exóticas Anglo-nubianas, 374 e 296

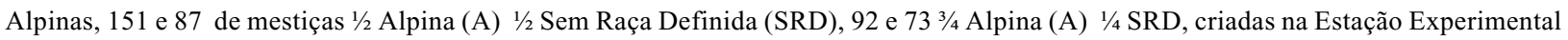
de Pendência - EMEPA-PB, Soledade-PB, na microrregião semi-árida da Paraíba, em regime semi-intensivo, no período de 1980 a 1994. $\mathrm{O}$ mês foi significativo sobre os pesos à cobrição das matrizes Canindés, Anglo-nubianas, $1 / 2 \mathrm{~A} \quad 1 / 2 \mathrm{SRD}$ e $3 / 4 \mathrm{~A} \quad 1 / 4 \mathrm{SRD}$. O ano influenciou o PC de todos os grupos genéticos. O efeito do ano sobre o GP foi significativo para as cabras Canindés, Anglo-nubianas, $1 / 2 \mathrm{~A} \quad 1 / 2 \mathrm{SRD}$ e Alpinas. O peso à cobrição elevou-se com o aumento da ordem de parto e o GP foi mais evidente entre as cabras de primeiro parto. $\mathrm{O}$ tipo de parto foi significativo sobre o GP das matrizes nubianas, 3/4 A 1/4 SRD e Alpina, que tiveram maiores ganhos quando pariram apenas um cabrito. A raça influenciou o PC e o GP de todas as matrizes. A raça nativa foi a mais leve e com o melhor ganho de peso; as exóticas, as mais pesadas e com GP inferiores aos da raça Canindé e da mestiça $1 \frac{1}{2} \mathrm{~A}$ 1/2 SRD; e a mestiça $3 / 4 \mathrm{~A}$ 1/4 SRD, a raça com peso corporal (PC) e GP semelhantes às exóticas. Concluiu-se que a composição genética dos animais e os fatores ambientais, como mês, ano, ordem e tipo de parto, refletiram sobre o peso à cobrição e o ganho de peso durante a gestação das matrizes estudadas.
\end{abstract}

Palavras-chave: caprinos, ganho de peso, gestação, peso à cobrição

\section{Mating Weight and Weight Gain During Gestation of Native, Exotic and Crossbred Goats in the Semi-Arid}

ABSTRACT - The effects of month, year, order and type parturition on mating weight (MW), weight gain during gestation (WG), respectively, of 753 and 527 Caninde native goats, 463 and 333 exotic Anglo Nubian and 374 and 296 Alpine goats, 151 and 87 crossbred 1/2 Alpine 1/2 SRD, and 92 and 73 3/4Alpine 1/4SRD goats, raised in the Experimental Station of the Governmental Research Institute (EMEPA-PB), Soledade - Paraiba - Brazil, in the period from 1980 to 1994. The effect of month was significant on $\mathrm{MW}$ of the Caninde, Anglo Nubian and crossbred goats. The year influenced MW of all genetic groups and effect on WG was significant for Caninde, Anglo Nubian, $1 / 2 \mathrm{~A} 1 / 2 \mathrm{SRD}$ and Alpine goats. Mating weight increased as parturition order increased and WG was evident in the goats of first kidding. Type parturition showed significant effect on WG of Nubian, $3 / 4$ A $1 / 4$ SRD and Alpine goats, that showed higher WG when had single kidding. Significant difference among genetic groups was observed for MW and WG. The native goats were more weightless than the exotic and crossbred goats, however showed better weight gain during gestation. There was superiority of mating weights of exotic than the native and crossbred goats. The animal genetic composition and environmental factors, as month, year, order and type of birth, affected mating weight and weight gain during the gestation of the studied flock.

Key Words: goats, gestation, mating weight, weight gain

\section{Introdução}

A avaliação do peso corporal de matrizes aptas à reprodução, como suporte para o planejamento do manejo reprodutivo, pode constituir uma forma eficiente no processo de seleção das matrizes a serem submetidas à cobrição.
Segundo Gonçalves (1996), como não é prática rotineira avaliar o peso dos animais nas criações comerciais, essas informações não estão disponíveis, e a maioria dos trabalhos relata o efeito da idade sobre as características produtivas.

Na região Nordeste, a época de cobrição das matrizes é fator determinante na produtividade do

\footnotetext{
${ }^{1}$ Parte da Dissertação de Mestrado apresentada ao CCA/UFPB pelo primeiro autor.

2 Professor da Escola Agrotécnica Federal de Codó-MA (geovergue@uol.com.br).

3 Professor do Centro de Ciências Agrárias/UFPB - Areia - PB (edgard@cca.ufpb.br).

${ }^{4}$ Pesquisador da Empresa Estadual de Pesquisa Agropecuária - EMEPA-PB (wandrick@emepa.org.br).
} 
rebanho, sendo importante coincidir a época de parição com o período de maior disponibilidade de alimentos na pastagem. Segundo Silva \& Silva (1987), na Bahia, quando as parições ocorrem na estação seca as cabras apresentam baixo desempenho reprodutivo pós-parto.

Chawla et al. (1982), Yappi et al. (1990) e Silveira et al. (1992) recomendam que a cobrição deve ser executada quando houver boa condição corporal do animal, para evitar abortos e mortalidade dos recém-nascidos.

Cabras fecundadas no início do período seco, podem perder peso no transcorrer da gestação. Essas perdas sugerem a necessidade de suplementação alimentar (Guimarães Filho, 1983; Araújo Filho et al., 1990). É fato comprovado o extremo decréscimo na qualidade e quantidade de forrageiras no período seco do ano, principalmente nas regiões semi-áridas (Schacht \& Malechek, 1989; Ramírez et al., 1991; Solanki, 1994).

Economides \& Louca (1987) e Oliveira (1992) recomendam suplementação com concentrados no período anterior à estação de cobrição, para que as fêmeas refaçam suas reservas orgânicas que foram consumidas durante a gestação e lactação anteriores, a fim de estimular aumento na taxa de ovulação.

Segundo Morand-Fehr \& Sauvant (1984), se as cabras estiverem gordas no período em que estão secas, deve ser fornecida forragem ad libitum com pouco concentrado, para evitar redução da taxa ovulatória no período de serviço. Recomendam, ainda, diminuição na alimentação suplementar depois da cobrição, para evitar o fracasso na sobrevivência embrionária.

A elevação do peso corporal durante a gestação de matrizes caprinas é mencionada por alguns autores. Ruvuna et al. (1991) verificaram em cabras East African e Galla que o peso corporal aumentou em decorrência da gestação (média de 6,2 e 6,0 kg para as duas raças, respectivamente).

Steine (1975) relatou regressão significativa do tipo de parto sobre o peso do corpo da cabra ao parto. Cabras que pariram dois ou três cabritos foram 0,5 e $2,0 \mathrm{~kg}$ mais pesadas, respectivamente, que cabras de parto simples.

Silva et al. (1986), trabalhando com ovinos, verificaram que os pesos pré-cobrição $(28,0$ e $29,7 \mathrm{~kg})$ e ao parto $(31,4$ e $31,6 \mathrm{~kg}$ ) para partos simples e múltiplos, respectivamente, não tiveram efeito significativo sobre o peso corporal pós-parto das matrizes, e reportaram que a perda de peso após o parto está associada ao peso da cria, placenta e líquidos, ao peso corporal e à idade da ovelha.

A ordem de parto constitui um importante fator de ambiente. Os processos de crescimento corporal e de elevação do peso corporal, geralmente, ocorrem em função da idade, quando atendidas as necessidades nutricionais. Segundo Sampaio et al. (1984), cabras jovens gestantes não têm seu crescimento prejudicado. Pelo contrário, seu desenvolvimento é estimulado, principalmente, quando estão submetidas a planos nutricionais adequados (Argheore et al., 1992).

O presente trabalho foi conduzido com o objetivo de avaliar os efeitos de mês, ano, ordem de parto, tipo de parto e grupo genético sobre os pesos à cobrição e o ganho de peso durante a gestação de cabras nativas, exóticas e mestiças, criadas no semi-árido paraibano.

\section{Material e Métodos}

O trabalho foi desenvolvido na Estação Experimental de Pendência, pertencente à Empresa Estadual de Pesquisa Agropecuária da Paraíba (EMEPA-PB), Soledade - PB, na microrregião semiárida do Cariri Ocidental, utilizando-se dados de pesos à cobrição e ganho de peso durante a gestação, respectivamente, de 753 e 527 cabras Canindés, pertencentes ao núcleo de preservação da raça; 463 e 333 Anglo-nubianas; 151 e 87 1/2 Alpina 1/2 SRD; 92 e 73 3/4 Alpina 1/4 SRD; e 374 e 296 Alpinas, no período de 1980 a 1994 .

Os animais foram criados em regime semiintensivo. Na estação chuvosa, a alimentação do rebanho foi constituída de pastagens nativas melhoradas com capim-buffel (Cenchrus ciliares L.), suplementação com concentrados para as lactantes e reprodutores e fornecimento de mistura mineral. $\mathrm{Na}$ época seca, além da pastagem, todos os animais receberam suplementação alimentar composta de capim-marrequinha (Howardia smilacina, Klotz), cunhã (Clitoria ternatea L.), leucena (Leucaena leucocephala), palma forrageira (Opuntia sp), silagem de milho, ração concentrada, mistura mineral, uréia e melaço.

Adotou-se o sistema de monta natural controlada, utilizando-se rufiões para detecção do cio. As estações de monta, geralmente, ocorreram nas épocas chuvosa (abril a junho) e seca (novembro a janeiro). Em alguns anos, as montas foram antecipadas ou retardadas, de acordo com a condição corporal das matrizes e a oferta de alimentos durante e após a gestação. As 
fêmeas jovens foram incorporadas ao rebanho de matrizes quando apresentaram peso igual ou superior a $65 \%$ da média de peso adulto das matrizes de cada grupo genético, contemporâneas do rebanho. O controle sanitário dos animais foi realizado por vacinações e combate aos endo e ectoparasitas. Os tratamentos contra querato-conjuntivite, linfadenite caseosa e do ectima contagioso foram adotados quando constatadas as ocorrências no rebanho.

$\mathrm{O}$ pequeno número de informações relativas às cabras com ordem de parto superior a cinco provocou sua exclusão das análises, em conseqüência do manejo reprodutivo adotado na Estação Experimental.

Para as análises, consideraram-se como fontes de variação os efeitos de mês e ano de cobrição, ordem de parição, tipo de parto e grupo genético.

Os pesos de machos e fêmeas dos rebanhos estudados eram mensurados a cada 28 dias. Realizaram-se, também, as pesagens das crias ao nascerem e das fêmeas logo após a cobertura e imediatamente após o parto, quando estas liberavam os envoltórios fetais. Estes restos placentários não foram mensurados.

O ganho de peso na gestação foi calculado a partir da diferença entre os pesos corporais registrados ao parto e ao acasalamento. Na seleção dos dados para o ganho de peso durante a gestação, consideraram-se os valores compreendidos no intervalo de - 8,0 a $8,0 \mathrm{~kg}$, para maior consistência das análises. $\mathrm{Na}$ análise de peso à cobrição, por razões óbvias, não foi considerado o tipo de parto. As análises de variância foram feitas pelo método dos Quadrados Mínimos e os testes de comparação de médias, pelo teste Tukey, a $\%$ de probabilidade, com o auxílio computacional do programa SAEG - Sistema de Análises Estatísticas e Genéticas (UFV, 1992).

Para as características de peso à cobrição por grupo genético, foi utilizado o modelo I.

$$
\mathrm{Y}_{\mathrm{ijk}}=\mu+\mathrm{MC}_{\mathrm{i}}+\mathrm{AC}_{\mathrm{j}}+\mathrm{OP}_{\mathrm{k}}+\varepsilon_{\mathrm{ijk}}
$$

em que $\mathrm{Y}_{\mathrm{ijk}}=$ peso médio à cobrição observado em cada cabra; $\mu$ = média geral da população; $\mathrm{MC}_{\mathrm{i}}=$ efeito do i-ésimo mês de cobrição, sendo $\mathrm{i}=1 . .6,11,12 ; \mathrm{AC}_{\mathrm{j}}=$ efeito do j-ésimo ano de cobrição, sendo $j=1981$...1994; $\mathrm{OP}_{\mathrm{k}}=$ efeito da k-ésima ordem de parto, sendo $\mathrm{k}=1 . . .5$; $\varepsilon_{\mathrm{ijk}}=$ erro aleatório associado a cada observação.

Para os pesos à cobrição entre grupo genético, utilizou-se o modelo II.

$$
\mathrm{Y}_{\mathrm{ijkl}}=\mu+\mathrm{RC}_{\mathrm{i}}+\mathrm{MC}_{\mathrm{j}}+\mathrm{AC}_{\mathrm{k}}+\mathrm{OPl}+\varepsilon_{\mathrm{ijkl}}
$$

em que $\mathrm{Y}_{\mathrm{ijkl}}=$ peso médio à cobrição observado em cada grupo genético; $\mu$ = média geral da população; $\mathrm{RC}_{\mathrm{i}}=$ efeito da i-ésima grupo genético; $\mathrm{MC}_{\mathrm{j}}=$ efeito do j-ésimo mês de cobrição, sendo $j=1 . . .7,9,11,12$; $\mathrm{AC}_{\mathrm{k}}=$ efeito do k-ésimo ano de cobrição, sendo $\mathrm{k}=1980 \ldots 1992,1994 ; \mathrm{OP}_{1}=$ efeito da l-ésimo ordem de parto, sendo $1=1 . .5 ; \varepsilon_{\mathrm{ijkl}}=$ erro aleatório associado a cada observação.

Na avaliação do ganho de peso durante a gestação por grupo genético, foi utilizado o modelo III.

$$
\mathrm{Y}_{\mathrm{ijkl}}=\mu+\mathrm{MC}_{\mathrm{i}}+\mathrm{AC}_{\mathrm{j}}+\mathrm{OP}_{\mathrm{k}}+\mathrm{TP}_{1}+\varepsilon_{\mathrm{ijk} \mathrm{l}}
$$

em que $Y_{\mathrm{ijkl}}=$ ganho de peso observado por cada cabra; $\mu=$ média geral da população; $\mathrm{MP}_{\mathrm{i}}=$ efeito do i-ésimo mês de cobrição, sendo $\mathrm{i}=1 \ldots 6,11,12 ; \mathrm{AP}_{\mathrm{j}}=$ efeito do j-ésimo ano de cobrição, sendo $\mathrm{j}=$ 1980...1992, 1994; $\mathrm{OP}_{\mathrm{k}}=$ efeito da k-ésima ordem de parto, sendo $\mathrm{k}=1 \ldots 5 ; \mathrm{TP}_{1}=$ efeito do 1-ésimo tipo de parto, sendo $1=1$ e $2 ; \varepsilon_{\mathrm{ijkl}}=$ erro aleatório associado a cada observação.

Para o ganho de peso durante a gestação entre os grupos genéticos, utilizou-se o modelo IV.

$$
\begin{gathered}
\mathrm{Y}_{\mathrm{ijklm}}=\mu+\mathrm{RC}_{\mathrm{i}}+\mathrm{MC}_{\mathrm{j}}+\mathrm{AC}_{\mathrm{k}}+\mathrm{OP}_{1}+\mathrm{TP}_{\mathrm{m}}+ \\
\mathrm{b}\left(\mathrm{X}_{\mathrm{ijklm}}-\mathrm{X}\right)+\varepsilon_{\mathrm{ijklm}}
\end{gathered}
$$

em que $Y_{\mathrm{ijklm}}=$ ganho de peso durante a gestação observado em cada grupo genético; $\mu=$ média geral da população; $\mathrm{RC}_{\mathrm{i}}=$ efeito da i-ésimo grupo genético; $\mathrm{MP}_{\mathrm{j}}=$ efeito do $\mathrm{j}$-ésimo mês de parto, sendo $\mathrm{j}=$ $2,4 \ldots 12 ; \mathrm{AP}_{\mathrm{k}}=$ efeito do k-ésimo ano de parto, sendo $\mathrm{k}=1980 \ldots 1995 ; \mathrm{OP}_{1}=$ efeito da l-ésima ordem de parto, sendo $1=1 \ldots 5 ; \mathrm{TP}_{\mathrm{m}}=$ efeito do m-ésimo tipo de parto, sendo $m=1$ e $2 ; b=$ coeficiente de regressão linear do ganho de peso em função do peso à cobrição; $\mathrm{X}_{\mathrm{ijklm}}=$ peso observado em cada grupo genético; $\varepsilon_{\mathrm{ijkl}}=$ erro aleatório associado a cada observação.

\section{Resultados e Discussão}

Observou-se influência $(\mathrm{P}<0,05)$ do mês sobre os pesos das matrizes. Os menores pesos à cobrição (Tabela 1) foram verificados nos meses de maior incidência de chuvas, fevereiro a abril, principalmente para as cabras Canindés, Anglo-nubianas, $1 / 2$ A $1 / 2$ 
SRD e $3 / 4$ A 1/4 SRD, quando registrou-se 24,57; 37,06; 29,48 e $32,91 \mathrm{~kg}$, respectivamente. Segundo Peter (1992), em condições de pastagem nativa da caatinga, os caprinos, por preferência alimentar, consomem maior quantidade de folhas de árvores, herbáceas e arbustos, em relação às gramíneas. Araújo Filho \& Silva (1994) reportam que na caatinga a fitomassa pastável de espécies lenhosas que participam ativamente da composição da dieta dos caprinos é baixa e que, quando o valor nutritivo e a digestibilidade são elevados, grande parte da folhagem produzida está fora do alcance dos animais.

Segundo Quittet (1978), quando a cobrição ocorre nos meses considerados chuvosos, momento em que há disponibilidade forrageira, verifica-se redução do peso da cabra, como reflexo do seu baixo consumo sob condições de elevada umidade do ambiente e da forragem.
Os pesos à cobrição das cabras cobertas nos meses finais da estação seca (novembro a janeiro), 29,76; 43,04; 33,60; 40,36 kg, para os grupos Canindé, Anglo-nubiana e $3 / 4$ A $1 / 4$ SRD, respectivamente, foram superiores aos da estação chuvosa (Tabela 1).

No entanto, na Tabela 2, observa-se que os ganhos de peso durante a gestação das matrizes cobertas no período de fevereiro a abril, principalmente das cabras Canindé, $1 / 2$ A $1 / 2$ SRD, $3 / 4$ A $1 / 4$ SRD e Alpina, que apresentaram médias no início da estação de 4,98; 4,15; 4,13; e 4,14 kg, respectivamente, foram maiores que os das matrizes cobertas na época seca (novembro a janeiro), de 0,$73 ; 1,51 ;-0,18 ; 0,50 \mathrm{~kg}$ para as nativas, nubianas, 3/4 A 1/4 SRD e alpinas, respectivamente, indicando que a gestação daquelas matrizes transcorreu em uma época de disponibilidade de alimentos na pastagem (fevereiro a setembro) e, conseqüentemente, houve consumo de alimentos

Tabela 1 - Médias de pesos à cobrição $(\mathrm{kg})$ de cabras nativas, exóticas e mestiças, em função do mês

Table 1 - Means of mating weight $(\mathrm{kg})$ of native, exotic and crossbred goats, in function of month

\begin{tabular}{|c|c|c|c|c|c|c|c|c|c|c|}
\hline \multirow{3}{*}{$\begin{array}{l}\text { Mês de } \\
\text { cobrição } \\
\text { Mating } \\
\text { month }\end{array}$} & \multicolumn{10}{|c|}{$\begin{array}{l}\text { Grupos genéticos } \\
\text { Genetic groups }\end{array}$} \\
\hline & \multicolumn{2}{|c|}{$\begin{array}{l}\text { Canindé } \\
\text { Caninde }\end{array}$} & \multicolumn{2}{|c|}{$\begin{array}{l}\text { Anglo-nubiana } \\
\text { Anglo Nubian }\end{array}$} & \multicolumn{2}{|c|}{$\begin{array}{l}1 / 2 \text { A } 1 / 2 \text { SRD } \\
1 / 2 A \text { I } 1 / 2 \text { SRD }\end{array}$} & \multicolumn{2}{|c|}{ 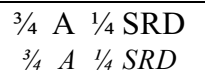 } & \multicolumn{2}{|c|}{$\begin{array}{l}\text { Alpina } \\
\text { Alpine }\end{array}$} \\
\hline & $\mathrm{N}^{\mathrm{o}}$ & $\begin{array}{l}\mathrm{PC}(\mathrm{kg}) \\
M W\end{array}$ & $\mathrm{~N}^{\mathrm{o}}$ & $\begin{array}{l}\mathrm{PC}(\mathrm{kg}) \\
M W\end{array}$ & $\mathrm{~N}^{\mathrm{o}}$ & $\begin{array}{l}\mathrm{PC}(\mathrm{kg}) \\
M W\end{array}$ & $\mathrm{~N}^{\circ}$ & $\begin{array}{l}\mathrm{PC}(\mathrm{kg}) \\
M W\end{array}$ & $\mathrm{~N}^{\mathrm{o}}$ & $\begin{array}{l}\mathrm{PC}(\mathrm{kg}) \\
M W\end{array}$ \\
\hline $\begin{array}{l}\text { JAN } \\
\text { Jan }\end{array}$ & 59 & $28,30 \mathrm{ab}$ & 53 & $39,39 b c$ & 20 & $33,60 \mathrm{a}$ & 11 & $40,36 \mathrm{a}$ & 42 & $37,81 \mathrm{a}$ \\
\hline $\begin{array}{l}\text { FEV } \\
\mathrm{Feb}\end{array}$ & 169 & $26,72 b$ & 27 & $37,03 \mathrm{c}$ & - & - & - & - & 17 & $40,82 \mathrm{a}$ \\
\hline $\begin{array}{l}\text { MAR } \\
\text { Mar }\end{array}$ & 218 & $24,99 \mathrm{c}$ & 86 & $38,27 \mathrm{c}$ & 38 & $33,28 \mathrm{a}$ & 23 & $32,91 \mathrm{~b}$ & 98 & $39,19 \mathrm{a}$ \\
\hline $\begin{array}{l}\mathrm{ABR} \\
\mathrm{Apr}\end{array}$ & 143 & $24,57 \mathrm{c}$ & 123 & $37,06 \mathrm{c}$ & 58 & $29,48 \mathrm{~b}$ & 27 & $34,37 \mathrm{~b}$ & 82 & $36,14 \mathrm{a}$ \\
\hline $\begin{array}{l}\text { MAI } \\
\text { May }\end{array}$ & 16 & $25,50 \mathrm{bc}$ & 31 & $42,74 \mathrm{ab}$ & 24 & $31,45 \mathrm{ab}$ & - & - & 37 & $38,94 \mathrm{a}$ \\
\hline $\begin{array}{l}\text { JUN } \\
\text { Jun }\end{array}$ & - & - & 42 & $43,14 \mathrm{a}$ & - & - & - & - & 41 & $36,46 \mathrm{a}$ \\
\hline $\begin{array}{l}\text { SET } \\
\text { Sept }\end{array}$ & - & - & - & - & 11 & $30,90 \mathrm{ab}$ & - & - & - & - \\
\hline $\begin{array}{l}\mathrm{NOV} \\
\text { Nov }\end{array}$ & 48 & $28,35 \mathrm{ab}$ & 44 & $43,04 \mathrm{a}$ & - & - & 12 & $37,50 \mathrm{ab}$ & 32 & $36,37 \mathrm{a}$ \\
\hline $\begin{array}{l}\text { DEZ } \\
\text { Dec }\end{array}$ & 100 & $29,76 \mathrm{a}$ & 57 & $41,42 \mathrm{ab}$ & - & - & 19 & $34,36 \mathrm{ab}$ & 25 & $39,68 \mathrm{a}$ \\
\hline $\begin{array}{l}\text { Médias } \\
\text { Means }\end{array}$ & 26,4 & $2 \pm 5,03 \mathrm{E}$ & 39,5 & $\pm 7,15 \mathrm{~A}$ & 31 , & $\pm 5,81 \mathrm{D}$ & 35 , & $\pm 6,59 \mathrm{C}$ & & $1 \pm 7,05 \mathrm{~B}$ \\
\hline
\end{tabular}

Médias seguidas de letras iguais, minúsculas, na coluna, e maiúsculas, na linha, não diferem a $5 \%$ pelo teste Tukey. Means followed by small and capital letters in the column and line, respectively, do not differ $(P<.05)$ by Tukey test.

$\mathrm{N}^{\circ}=$ Número de dados observados.

$N=$ Number of observations.

$\mathrm{PC}=$ Peso à cobrição.

$M W=$ Mating weight.

R. Bras. Zootec., v.33, n.6, p.1711-1720, 2004 (Supl. 1) 
Tabela 2 - Médias de ganho de peso $(\mathrm{kg})$ durante a gestação de cabras nativas, exóticas e mestiças, em função do mês

Table 2 - Means of weight gain during gestation of native, exotic and crossbred goats, in function of month

\begin{tabular}{|c|c|c|c|c|c|c|c|c|c|c|}
\hline \multirow{3}{*}{$\begin{array}{l}\text { Mês de } \\
\text { cobrição } \\
\text { Mating } \\
\text { month }\end{array}$} & \multicolumn{10}{|c|}{$\begin{array}{l}\text { Grupos genéticos } \\
\text { Genetic groups }\end{array}$} \\
\hline & \multicolumn{2}{|c|}{$\begin{array}{l}\text { Canindé } \\
\text { Caninde }\end{array}$} & \multicolumn{2}{|c|}{$\begin{array}{l}\text { Anglo-nubiana } \\
\text { Anglo Nubian }\end{array}$} & \multicolumn{2}{|c|}{$\begin{array}{l}1 / 2 \text { A } 1 / 2 \text { SRD } \\
1 / 2 A 1 / 2 S R D\end{array}$} & \multicolumn{2}{|c|}{$\begin{array}{l}3 / 4 \text { A } 1 / 4 \text { SRD } \\
3 / 4 \text { A } 1 / 4 \text { SRD }\end{array}$} & \multicolumn{2}{|c|}{$\begin{array}{l}\text { Alpina } \\
\text { Alpine }\end{array}$} \\
\hline & $\mathrm{N}^{\mathrm{o}}$ & $\begin{array}{l}\mathrm{GP}(\mathrm{kg}) \\
W G\end{array}$ & $\mathrm{~N}^{\mathrm{o}}$ & $\begin{array}{l}\mathrm{GP}(\mathrm{kg}) \\
W G\end{array}$ & $\mathrm{~N}^{\circ}$ & $\begin{array}{l}\mathrm{GP}(\mathrm{kg}) \\
W G\end{array}$ & $\mathrm{~N}^{\mathrm{o}}$ & $\begin{array}{l}\mathrm{GP}(\mathrm{kg}) \\
W G\end{array}$ & $\mathrm{~N}^{\mathrm{o}}$ & $\begin{array}{l}\text { GP }(\mathrm{kg}) \\
W G\end{array}$ \\
\hline $\begin{array}{l}\text { JAN } \\
\text { Jan }\end{array}$ & 49 & $3,61 \mathrm{~b}$ & 41 & $4,90 \mathrm{a}$ & 12 & $4,50 \mathrm{a}$ & - & - & 34 & $2,85 \mathrm{ab}$ \\
\hline $\begin{array}{l}\text { FEV } \\
\mathrm{Feb}\end{array}$ & 128 & $4,35 \mathrm{ab}$ & 18 & $3,61 \mathrm{ab}$ & - & - & - & - & 21 & $4,14 \mathrm{a}$ \\
\hline $\begin{array}{l}\text { MAR } \\
\text { Mar }\end{array}$ & 127 & $4,98 \mathrm{a}$ & 67 & $1,74 \mathrm{bc}$ & 25 & $2,08 \mathrm{~b}$ & 23 & $3,04 \mathrm{ab}$ & 95 & $2,95 \mathrm{ab}$ \\
\hline $\begin{array}{l}\mathrm{ABR} \\
\mathrm{Apr}\end{array}$ & 88 & $4,11 \mathrm{ab}$ & 84 & $2,86 \mathrm{~b}$ & 32 & $4,15 \mathrm{a}$ & 23 & $4,13 \mathrm{a}$ & 64 & $3,78 \mathrm{a}$ \\
\hline $\begin{array}{l}\text { MAI } \\
\text { May }\end{array}$ & - & - & 20 & $2,80 \mathrm{abc}$ & 18 & $2,38 \mathrm{ab}$ & - & - & 32 & $3,56 \mathrm{a}$ \\
\hline $\begin{array}{l}\text { JUN } \\
\text { Jun }\end{array}$ & - & - & 28 & $0,46 \mathrm{c}$ & - & - & - & - & - & - \\
\hline $\begin{array}{l}\text { NOV } \\
\text { Nov }\end{array}$ & 41 & $0,73 \mathrm{c}$ & 30 & $2,86 \mathrm{abc}$ & - & - & 11 & $-0,18 \mathrm{c}$ & 24 & $0,50 \mathrm{~b}$ \\
\hline $\begin{array}{l}\text { DEZ } \\
\text { Dec }\end{array}$ & 84 & $1,01 \mathrm{c}$ & 45 & $1,51 \mathrm{bc}$ & - & - & 16 & $1,81 \mathrm{bc}$ & 26 & $1,07 \mathrm{ab}$ \\
\hline Médias & 3,5 & $\pm 3,26 \mathrm{~A}$ & 2,54 & $\pm 3,77 \mathrm{~B}$ & 3,24 & $=3,10 \mathrm{AB}$ & 2,63 & $\pm 3,80 \mathrm{~B}$ & 2,9 & $\pm 3,97 \mathrm{~B}$ \\
\hline
\end{tabular}

Means

Médias seguidas de letras iguais, minúsculas, na coluna, e maiúsculas, na linha, não diferem a $5 \%$ pelo teste Tukey. Means followed by small and capital letters in the column and line, respectively, do not differ $(P<.05)$ by Tukey test. $\mathrm{N}^{\circ}=$ Número de dados observados

$N=$ Number of observations.

GP = Ganho em peso durante a gestação

$W G=$ Weight gain during gestation .

capaz de promover ganhos de peso, contrariando a informação de Quittet (1978).

Na Figura 1, observa-se a variação dos pesos à cobrição das matrizes de ano para ano. As cabras Canindé apresentaram baixos pesos à cobrição nos anos de $1984(22,61 \mathrm{~kg})$ e de $1987(23,78 \mathrm{~kg})$. Esses pesos podem ser atribuídos a um razoável número de matrizes jovens selecionadas para reprodução, além do manejo que era diferenciado, pois as cabras Canindé eram criadas quase que extensivamente. A partir de 1989, estas cabras apresentaram maiores pesos, permanecendo acima da média, o que se deve ao aumento da idade e de sua inclusão no programa de melhoramento genético, quando passaram a receber manejo mais adequado e semelhante ao dos outros grupos genéticos.

As cabras 3/4 A 1/4 SRD apresentaram menor peso à cobrição $(31,26 \mathrm{~kg}), \mathrm{em} 1988$, por constituírem um rebanho recém-formado de matrizes com esta composição genética.
O ganho de peso das cabras Canindé até 1988 manteve-se sempre acima da média desta raça, principalmente em 1987 (5,54 kg) (Tabela 3). Em 1992, o ganho foi muito reduzido, ficando em torno de $0,56 \mathrm{~kg}$. As cabras Anglo-nubianas obtiveram ganho de peso de 6,80, em 1981, e de $-2,69 \mathrm{~kg}$, em 1983. As mestiças 1/2 A $1 / 2 \mathrm{SRD}$, em 1987, apresentaram o menor ganho (1,66 kg), que, em 1988, aumentou para 4,23 kg. Para as matrizes Alpinas, registrou-se ganho de 6,21 kg, em 1980, época em que foram importadas da Alemanha e, portanto, receberam manejo diferenciado, e de -0,36 kg, em 1992. Fatores como incidência e regularidade das chuvas, entre os anos do período considerado, idade e manejo alimentar dos animais podem ter contribuído para os ganhos durante a gestação.

Os pesos à cobrição apresentaram tendência de aumento com o incremento da ordem de parição (Tabela 4).

Nota-se que os pesos das cabras submetidas à primeira cobrição representam, para a Canindé, Anglo- 


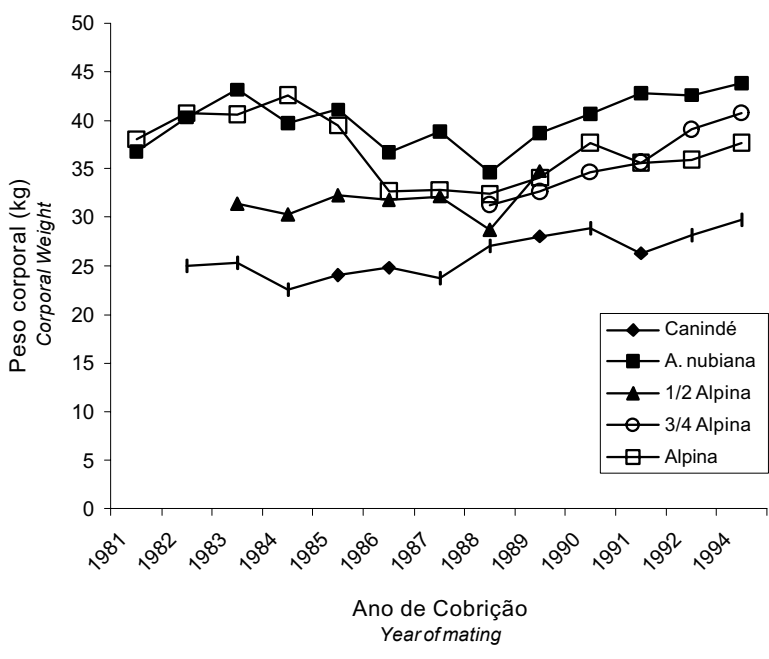

Figura 1 - Variações dos pesos à cobrição de cabras nativas, exóticas e mestiças, em função do ano.

Figure 1 - Variations of mating weights of native, exotic and crossbred goats, in function of the year. nubiana, $1 / 2$ A $1 / 2 \mathrm{SRD} \mathrm{e}^{3 / 4} \mathrm{~A}$ 1/4 SRD e Alpina, 78; 77,2; 80,$86 ; 86,8$ e $79,2 \%$, respectivamente, em relação à média de peso das cabras de quinta parição de cada grupo genético. Isto sugere que o critério de peso adotado para o primeiro acasalamento, mesmo em condições de semi-árido, está retardando o início da vida reprodutiva dessas matrizes, o que implica em elevada idade ao primeiro parto e maiores custos com a criação das fêmeas jovens e, ainda, menor longevidade.

Gonçalves (1996) reporta que o alto valor para a idade ao primeiro parto pode ser reflexo da falta de um manejo adequado para as cabritas em crescimento ou do critério de peso utilizado para a primeira cobrição (30-35 kg), que considera elevado para as cabras nas condições brasileiras, visto que este valor se baseia em uma porcentagem do peso adulto dos animais nas condições de clima temperado.

O ganho de peso foi mais evidente nas cabras de primeira parição, 3,$87 ; 4,45 ;$ e $3,50 \mathrm{~kg}$ para as matrizes Canindés, $1 / 2$ A $1 / 2$ SRD e Alpina, respectivamente

Tabela 3 - Médias de ganho de peso $(\mathrm{kg})$ durante a gestação de cabras nativas, exóticas e mestiças, em função do ano

Table 3 - Means of weight gain during gestation of native, exotic and crossbred goats, according to the year

\begin{tabular}{|c|c|c|c|c|c|c|c|c|c|c|}
\hline \multirow{3}{*}{$\begin{array}{l}\text { Ano de } \\
\text { cobrição } \\
\text { Mating } \\
\text { year }\end{array}$} & \multicolumn{10}{|c|}{$\begin{array}{l}\text { Grupos genéticos } \\
\text { Genetic groups }\end{array}$} \\
\hline & \multicolumn{2}{|c|}{$\begin{array}{l}\text { Canindé } \\
\text { Caninde }\end{array}$} & \multicolumn{2}{|c|}{$\begin{array}{l}\text { Anglo-nubiana } \\
\text { Anglo Nubian }\end{array}$} & \multicolumn{2}{|c|}{$\begin{array}{ccc}1 / 2 & \mathrm{~A} & 1 / 2 \\
1 / 2 & \mathrm{SRD} \\
1 / 2 & S R D\end{array}$} & \multicolumn{2}{|c|}{ 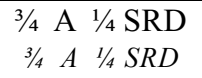 } & \multicolumn{2}{|c|}{$\begin{array}{l}\text { Alpina } \\
\text { Alpine }\end{array}$} \\
\hline & $\mathrm{N}^{\circ}$ & $\begin{array}{c}\mathrm{GP}(\mathrm{kg}) \\
W G\end{array}$ & $\mathrm{~N}^{\mathrm{o}}$ & $\begin{array}{c}\mathrm{GP}(\mathrm{kg}) \\
W G\end{array}$ & $\mathrm{~N}^{\circ}$ & $\begin{array}{c}\mathrm{GP}(\mathrm{kg}) \\
W G\end{array}$ & $\mathrm{~N}^{\mathrm{o}}$ & $\begin{array}{c}\mathrm{GP}(\mathrm{kg}) \\
W G\end{array}$ & $\mathrm{~N}^{\mathrm{o}}$ & $\begin{array}{c}\mathrm{GP}(\mathrm{kg}) \\
W G\end{array}$ \\
\hline 1980 & - & - & 15 & $1,46 \mathrm{bcd}$ & - & - & - & - & 28 & $6,21 \mathrm{a}$ \\
\hline 1981 & - & - & 10 & $6,80 \mathrm{a}$ & - & - & - & - & 27 & $3,55 \mathrm{abc}$ \\
\hline 1982 & 29 & $5,87 \mathrm{a}$ & 19 & $4,63 \mathrm{abc}$ & - & - & - & - & 31 & $4,77 \mathrm{ab}$ \\
\hline 1983 & 25 & $4,76 \mathrm{a}$ & 13 & $-2,69 \mathrm{e}$ & - & - & - & - & 14 & $2,07 \mathrm{bc}$ \\
\hline 1984 & - & - & 22 & $4,68 \mathrm{ab}$ & - & - & - & - & 35 & $1,60 \mathrm{c}$ \\
\hline 1985 & 67 & $5,51 \mathrm{a}$ & 23 & $2,30 \mathrm{bcd}$ & 15 & $3,80 \mathrm{ab}$ & - & - & 27 & $1,55 \mathrm{c}$ \\
\hline 1986 & 43 & $4,09 \mathrm{ab}$ & 21 & $3,09 \mathrm{bcd}$ & - & - & - & - & 20 & $2,80 \mathrm{abc}$ \\
\hline 1987 & 41 & $5,54 \mathrm{a}$ & - & - & 18 & $1,66 \mathrm{c}$ & - & - & - & - \\
\hline 1988 & 19 & $5,31 \mathrm{a}$ & 37 & $1,02 \mathrm{~d}$ & 27 & $4,23 \mathrm{a}$ & 13 & $4,77 \mathrm{a}$ & 19 & $3,47 \mathrm{abc}$ \\
\hline 1989 & 63 & $2,71 \mathrm{bc}$ & 62 & $2,81 \mathrm{bcd}$ & 27 & $2,60 \mathrm{bc}$ & 22 & $2,72 \mathrm{a}$ & 16 & $3,12 \mathrm{abc}$ \\
\hline 1990 & 95 & $2,04 \mathrm{~cd}$ & 55 & $1,82 \mathrm{~cd}$ & - & - & 15 & $2,20 \mathrm{a}$ & 33 & $2,24 \mathrm{bc}$ \\
\hline 1991 & 53 & $2,58 \mathrm{bc}$ & 12 & $3,00 \mathrm{abcd}$ & - & - & 10 & $2,00 \mathrm{a}$ & 18 & $2,39 \mathrm{bc}$ \\
\hline 1992 & 46 & $0,56 \mathrm{~d}$ & 25 & $2,44 \mathrm{bcd}$ & - & - & 13 & $1,31 \mathrm{a}$ & 14 & $-0,36 c$ \\
\hline 1993 & - & - & - & - & - & - & - & - & - & - \\
\hline 1994 & 36 & $4,33 \mathrm{ab}$ & 19 & $3,89 \mathrm{abcd}$ & - & - & - & - & 14 & $2,28 \mathrm{abc}$ \\
\hline $\begin{array}{l}\text { Médias } \\
\text { Means }\end{array}$ & \multicolumn{2}{|c|}{$3,56 \pm 3,26 \mathrm{~A}$} & \multicolumn{2}{|c|}{$2,54 \pm 3,77 \mathrm{~B}$} & \multicolumn{2}{|c|}{$3,24 \pm 3,10 \mathrm{AB}$} & \multicolumn{2}{|c|}{$2,63 \pm 3,80 \mathrm{~B}$} & \multicolumn{2}{|c|}{$2,91 \pm 3,97 \mathrm{~B}$} \\
\hline
\end{tabular}

R. Bras. Zootec., v.33, n.6, p.1711-1720, 2004 (Supl. 1) 
Tabela 4 - Médias de pesos à cobrição de cabras nativas, exóticas e mestiças, em função da ordem de parto

Table 4 - Means of mating weight $(\mathrm{kg})$ of native, exotic and crossbred goats, according to the parturition order

\begin{tabular}{|c|c|c|c|c|c|c|c|c|c|c|}
\hline \multirow{3}{*}{$\begin{array}{l}\text { Ordem } \\
\text { de parto } \\
\text { Parturition } \\
\text { order }\end{array}$} & \multicolumn{10}{|c|}{$\begin{array}{l}\text { Grupos genéticos } \\
\text { Genetic groups }\end{array}$} \\
\hline & \multicolumn{2}{|c|}{$\begin{array}{l}\text { Canindé } \\
\text { Caninde }\end{array}$} & \multicolumn{2}{|c|}{$\begin{array}{l}\text { Anglo-nubiana } \\
\text { Anglo Nubian }\end{array}$} & \multicolumn{2}{|c|}{$\begin{array}{l}1 / 2 \text { A } 1 / 2 \text { SRD } \\
1 / 2 A \text { A } 1 / 2 \text { SRD }\end{array}$} & \multicolumn{2}{|c|}{ 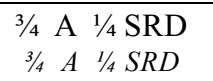 } & \multicolumn{2}{|c|}{$\begin{array}{l}\text { Alpina } \\
\text { Alpine }\end{array}$} \\
\hline & № & $\begin{array}{c}\mathrm{PC}(\mathrm{kg}) \\
M W \\
\end{array}$ & № & $\begin{array}{c}\mathrm{PC}(\mathrm{kg}) \\
M W \\
\end{array}$ & № & $\begin{array}{c}\mathrm{PC}(\mathrm{kg}) \\
M W \\
\end{array}$ & № & $\begin{array}{c}\mathrm{PC}(\mathrm{kg}) \\
M W \\
\end{array}$ & № & $\begin{array}{c}\mathrm{PC}(\mathrm{kg}) \\
M W \\
\end{array}$ \\
\hline 1 & 276 & $23,95 \mathrm{~d}$ & 163 & $35.36 \mathrm{c}$ & 75 & $28,77 b$ & 43 & 32,74 & 95 & $33,82 \mathrm{~d}$ \\
\hline 2 & 207 & 26 , & 132 & 4 & 42 & $32,85 \mathrm{a}$ & 31 & $36,93 \mathrm{a}$ & 108 & $2 \mathrm{c}$ \\
\hline 3 & 152 & 28 & 97 & 4 & 22 & $35,31 \mathrm{a}$ & 18 & $37,72 \mathrm{a}$ & 81 & 441 \\
\hline 4 & 80 & $29,47 \mathrm{ab}$ & 51 & 45 & 12 & $35,58 \mathrm{a}$ & - & - & 56 & $41,23 \mathrm{ab}$ \\
\hline$\underline{5}$ & 38 & $30,73 \mathrm{a}$ & 20 & $45,80 \mathrm{a}$ & - & - & - & - & 34 & $42,70 \mathrm{a}$ \\
\hline dias & 26 & $+5,03 \mathrm{E}$ & 39,5 & $7,15 \mathrm{~A}$ & 31,4 & 81 & 35,1 & $\mathrm{C}$ & & $1+7,04$ \\
\hline
\end{tabular}

Means

Médias seguidas de letras iguais, minúsculas, na coluna, e maiúsculas, na linha, não diferem a $5 \%$ pelo teste Tukey. Means followed by small and capital letters in the column and line, respectively, do not differ $(P<.05)$ by Tukey test. № $=$ Número de dados observados .

$N=$ Number of observations.

$\mathrm{PC}=$ Peso à cobrição.

$M W=$ Mating weight.

(Tabela 5). Sampaio et al. (1984) e Anderson (1986) comentam que, quando as exigências nutricionais são atendidas, a gestação beneficia o crescimento do feto, incrementa o peso corporal da mãe e até estimula o desenvolvimento das matrizes jovens.

Fehr (1981) reporta ganho real de peso, durante primeira gestação, em torno de $12,0 \mathrm{~kg}$. Na primeira lactação, o peso corporal aumentou de 10,0 a $14,0 \mathrm{~kg}$ $\mathrm{e}$, durante o segundo ciclo reprodutivo (gestaçãolactação), o ganho foi menor (2,0 a 7,0 kg) em relação ao peso à primeira cobrição.

$\mathrm{O}$ tipo de parto influenciou $(\mathrm{P}<0,05)$ o ganho de peso das cabras Anglo-nubianas, $1 / 2$ A $1 / 2$ SRD e Alpinas. As matrizes que tiveram partos múltiplos apresentaram ganhos de peso em torno de 1,$83 ; 2,76$ e $2,33 \mathrm{~kg}$, respectivamente, inferiores $(41,15 ; 8,31 \mathrm{e}$ $37,36 \%$ ) aos registrados para as cabras de partos simples (Tabela 6). Este fato pode estar associado à idade, pois as cabras de partos simples eram jovens, principalmente nulíparas e primíparas em crescimento, portanto, com o trato reprodutivo ainda em desenvolvimento (Awemu et al., 1999; Silva \& Araújo, 2000), bem como ao aumento das exigências nutricionais para manutenção da gestação dupla, associada à deficiência de nutrientes na alimentação com a conseqüente mobilização de reservas.

Esses resultados diferem dos encontrados por Steine (1975), que observou maior ganho de peso
$(0,5$ a $2,0 \mathrm{~kg})$ para as cabras de partos múltiplos em relação às cabras com gestação simples.

$\mathrm{Na}$ Tabela 7, observa-se que houve influência $(\mathrm{P}<0,05)$ da raça sobre o peso à cobrição. As matrizes Canindés se apresentaram as mais leves $(26,42 \pm$ $5,03 \mathrm{~kg}$ ), ratificando a informação de Figueiredo \& Pant (1982), de que os animais nativos encontrados no Nordeste são de porte hipométrico e apresentam peso adulto em torno de $30 \mathrm{a} 40 \mathrm{~kg}$.

Entre as matrizes de raças exóticas, as Anglonubianas foram as que evidenciaram maiores pesos à cobrição $(39,59 \pm 7,15 \mathrm{~kg})$, seguida das Alpinas $(37,91 \pm 7,05 \mathrm{~kg})$, por serem animais de porte elevado. Shelton (1978) e Majid et al. (1993), nos Estados Unidos, citam pesos adultos das duas raças em torno de 64 e 71,5 kg, para as cabras Anglo-nubianas, e de 50 e $64,8 \mathrm{~kg}$, para as Alpinas.

As matrizes mestiças apresentaram médias de pesos à cobrição intermediárias entre a nativa $\mathrm{e}$ as raças exóticas $(31,40 \pm 5,81$ e $35,13 \pm 6,59 \mathrm{~kg})$, para $1 / 2$ A $1 / 2$ SRD e $3 / 4$ A $1 / 4$ SRD, respectivamente. Nota-se, ainda, que o aumento na participação genética de Alpinas incrementou o peso corporal. Resultados similares foram encontrados por Pu et al. (1987), na China, e Jagtap et al. (1991), na Índia, quando cruzaram cabras nativas com machos de raças exóticas.

$\mathrm{O}$ efeito de grupo genético foi significativo $(\mathrm{P}<0,05)$ sobre o ganho de peso durante a gestação

R. Bras. Zootec., v.33, n.6, p.1711-1720, 2004 (Supl. 1) 
Tabela 5 - Médias de ganho de peso $(\mathrm{kg})$ durante a gestação de cabras nativas, exóticas e mestiças, em função da ordem de parto

Table 5 - Means of gain weight $(\mathrm{kg})$ during gestation of native, exotic and crossbred goats, according to the parturition order

\begin{tabular}{|c|c|c|c|c|c|c|c|c|c|c|}
\hline \multirow{3}{*}{$\begin{array}{l}\text { Ordem } \\
\text { de parto } \\
\text { Parturition } \\
\text { order }\end{array}$} & \multicolumn{10}{|c|}{$\begin{array}{c}\text { Grupos genéticos } \\
\text { Genetic groups }\end{array}$} \\
\hline & \multicolumn{2}{|c|}{$\begin{array}{l}\text { Canindé } \\
\text { Caninde }\end{array}$} & \multicolumn{2}{|c|}{$\begin{array}{l}\text { Anglo-nubiana } \\
\text { Anglo Nubian }\end{array}$} & \multicolumn{2}{|c|}{$\begin{array}{l}1 / 2 \text { A } 1 / 2 \text { SRD } \\
1 / 2 A \text { A } 1 / 2 \text { SRD }\end{array}$} & \multicolumn{2}{|c|}{ 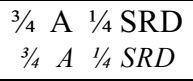 } & \multicolumn{2}{|c|}{$\begin{array}{l}\text { Alpina } \\
\text { Alpine }\end{array}$} \\
\hline & № & $\begin{array}{c}\mathrm{GP}(\mathrm{kg}) \\
\quad W G\end{array}$ & № & $\begin{array}{c}\mathrm{GP}(\mathrm{kg}) \\
W G\end{array}$ & № & $\begin{array}{c}\mathrm{GP}(\mathrm{kg}) \\
\quad W G\end{array}$ & № & $\begin{array}{c}\mathrm{GP}(\mathrm{kg}) \\
W G\end{array}$ & № & $\begin{array}{c}\mathrm{GP}(\mathrm{kg}) \\
W G\end{array}$ \\
\hline 1 & 197 & $3,87 \mathrm{a}$ & 129 & $2,32 \mathrm{a}$ & 38 & $4,45 \mathrm{a}$ & 40 & $2,67 \mathrm{a}$ & 103 & $3,49 \mathrm{a}$ \\
\hline 2 & 145 & $3,55 \mathrm{ab}$ & 101 & $2,98 \mathrm{a}$ & 25 & $2,52 \mathrm{~b}$ & 22 & $3,18 \mathrm{a}$ & 76 & $3,30 \mathrm{ab}$ \\
\hline 3 & 91 & $3,30 a b$ & 58 & $2,12 \mathrm{a}$ & 13 & $2,61 \mathrm{ab}$ & 11 & $1,36 \mathrm{a}$ & 56 & $2,37 \mathrm{ab}$ \\
\hline 4 & 53 & $3,73 \mathrm{ab}$ & 33 & $3,42 \mathrm{a}$ & 11 & $1,45 \mathrm{~b}$ & - & - & 38 & $2,45 a b$ \\
\hline 5 & 31 & $2,19 \mathrm{~b}$ & 12 & $0,92 \mathrm{a}$ & - & - & - & - & 23 & $1,04 \mathrm{~b}$ \\
\hline $\begin{array}{l}\text { Médias } \\
\text { Means }\end{array}$ & 3,56 & $3,26 \mathrm{~A}$ & 2,54 & $3,77 \mathrm{~B}$ & 3,24 & $3,10 \mathrm{AB}$ & 2,6 & $3,80 \mathrm{~B}$ & & $\pm 3,97 \mathrm{~B}$ \\
\hline
\end{tabular}

Médias seguidas de letras iguais, minúsculas, na coluna, e maiúsculas, na linha, não diferem a $5 \%$ pelo teste Tukey. Means followed by small and capital letters in the column and line, respectively, do not differ $(P<.05)$ by Tukey test. № = Número de dados observados.

$N=$ Number of observations.

$\mathrm{GP}=$ Ganho em peso durante a gestação

$W G=$ Weight gain during gestation .

Tabela 6 - Médias de ganho de peso $(\mathrm{kg})$ durante a gestação de cabras nativas, exóticas e mestiças, em função do tipo de parto

Table 6 - Means of weight gain during gestation of native, exotic and crossbred goats, according to the parturition type

\begin{tabular}{|c|c|c|c|c|c|c|c|c|c|c|}
\hline \multirow{3}{*}{$\begin{array}{l}\text { Tipo } \\
\text { de parto } \\
\text { Parturition } \\
\text { type }\end{array}$} & \multicolumn{10}{|c|}{$\begin{array}{l}\text { Grupos genéticos } \\
\text { Genetic groups }\end{array}$} \\
\hline & \multicolumn{2}{|c|}{$\begin{array}{l}\text { Canindé } \\
\text { Caninde }\end{array}$} & \multicolumn{2}{|c|}{$\begin{array}{l}\text { Anglo-nubiana } \\
\text { Anglo Nubian }\end{array}$} & \multicolumn{2}{|c|}{$\begin{array}{l}1 / 2 \text { A } 1 / 2 \text { SRD } \\
1 / 2 A \text { 1/2 SRD }\end{array}$} & \multicolumn{2}{|c|}{ 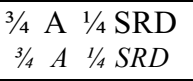 } & \multicolumn{2}{|c|}{$\begin{array}{l}\text { Alpina } \\
\text { Alpine }\end{array}$} \\
\hline & № & $\begin{array}{c}\mathrm{GP}(\mathrm{kg}) \\
W G\end{array}$ & № & $\begin{array}{c}\mathrm{GP}(\mathrm{kg}) \\
W G\end{array}$ & № & $\begin{array}{c}\mathrm{GP}(\mathrm{kg}) \\
W G\end{array}$ & № & $\begin{array}{c}\mathrm{GP}(\mathrm{kg}) \\
W G\end{array}$ & № & $\begin{array}{c}\mathrm{GP}(\mathrm{kg}) \\
W G \\
\end{array}$ \\
\hline $\begin{array}{l}\text { Simples } \\
\text { Single }\end{array}$ & 312 & $3,72 \mathrm{a}$ & 185 & $3,11 \mathrm{a}$ & 57 & $3,01 \mathrm{a}$ & 48 & $2,64 \mathrm{a}$ & 172 & $3,72 \mathrm{a}$ \\
\hline $\begin{array}{l}\text { Duplo } \\
\text { Twin } \\
\end{array}$ & 205 & $3,33 \mathrm{a}$ & 148 & $1,83 \mathrm{~b}$ & 30 & $2,76 \mathrm{~b}$ & 25 & $2,60 \mathrm{a}$ & 124 & $2,33 \mathrm{~b}$ \\
\hline $\begin{array}{l}\text { Médias } \\
\text { Means }\end{array}$ & \multicolumn{2}{|c|}{$3,56 \pm 3,26 \mathrm{~A}$} & \multicolumn{2}{|c|}{$2,54 \pm 3,77 \mathrm{~B}$} & \multicolumn{2}{|c|}{$3,24 \pm 3,10 \mathrm{AB}$} & \multicolumn{2}{|c|}{$2,63 \pm 3,80 \mathrm{~B}$} & \multicolumn{2}{|c|}{$2,91 \pm 3,97 \mathrm{~B}$} \\
\hline \multicolumn{11}{|c|}{$\begin{array}{l}\text { Médias seguidas de letras iguais, minúsculas, na coluna, e maiúsculas, na linha, não diferem a } 5 \% \text { pelo teste Tukey } \\
\text { Means followed by small and capital letters in the column and line, respectively, do not differ }(P<.05) \text { by Tukey test. } \\
\mathrm{N}^{\circ}=\text { Número de dados observados. } \\
N=\text { Number of observations. } \\
\text { GP = Ganho em peso durante a gestação. } \\
\text { WG = Weight gain during gestation. }\end{array}$} \\
\hline
\end{tabular}


Tabela 7 - Médias de peso à cobrição e ganho de peso $(\mathrm{kg})$ durante a gestação, em função do grupo racial

Table 7 - Means of mating weight and weight gain during gestation, according to the racial group

\begin{tabular}{|c|c|c|c|c|c|}
\hline & & & $\begin{array}{c}\text { Grupos genéticos } \\
\text { Genetic groups }\end{array}$ & & \\
\hline & $\begin{array}{l}\text { Canindé } \\
\text { Caninde }\end{array}$ & $\begin{array}{l}\text { Anglo-nubiana } \\
\text { Anglo Nubian }\end{array}$ & 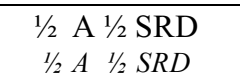 & $\begin{array}{ccc}3 / 4 & \mathrm{~A} & 1 / 4 \\
3 / 4 & A & \text { SRD } \\
1 / 4 & S R D\end{array}$ & $\begin{array}{l}\text { Alpina } \\
\text { Alpine }\end{array}$ \\
\hline $\begin{array}{l}\overline{\mathrm{PC}(\mathrm{kg})} \\
M W\end{array}$ & $26,42 \pm 5,03 \mathrm{E}$ & $39,59 \pm 7,15 \mathrm{~A}$ & $31,40 \pm 5,81 \mathrm{D}$ & $35,13 \pm 6,59 \mathrm{C}$ & $37,91 \pm 7,04 \mathrm{~B}$ \\
\hline $\begin{array}{l}\mathrm{GP}(\mathrm{kg}) \\
W G\end{array}$ & $3,56 \pm 3,26 \mathrm{~A}$ & $2,54 \pm 3,77 \mathrm{~B}$ & $3,24 \pm 3,10 \mathrm{AB}$ & $2,63 \pm 3,80 \mathrm{~B}$ & $2,91 \pm 3,97 \mathrm{~B}$ \\
\hline
\end{tabular}

(Tabela 7). As cabras Canindés apresentaram os melhores ganhos durante a gestação $(3,56 \pm 3,26 \mathrm{~kg})$, seguidas das mestiças $1 / 2 \mathrm{~A} 1 / 2 \mathrm{SRD}(3,24 \pm 3,80 \mathrm{~kg})$. As cabras Anglo-nubianas, Alpinas e $3 / 4 \mathrm{~A} 1 / 4 \mathrm{SRD}$ tiveram ganhos de peso semelhantes, $2,54 \pm 3,77$; $2,63 \pm 3,80 ; 2,91 \pm 3,97 \mathrm{~kg}$, respectivamente.

As cabras Canindés, mais rústicas, adaptam-se melhor ao ecossistema do semi-árido e apresentam-se como matrizes de baixa produção de leite; conseqüentemente, menos exigentes em termos nutricionais. Talvez essas características expliquem a maior capacidade de ganhar peso durante a gestação em relação às cabras dos outros grupos genéticos de maior potencial leiteiro.

\section{Conclusões}

A composição genética dos animais e, principalmente, os fatores ambientais, como mês, ano, ordem e tipo de parto, refletiram sobre o peso à cobrição e o ganho de peso durante a gestação das matrizes estudadas, fatores que estão relacionados à idade das cabras, ao número de crias por gestação, percentual de cabras nulíparas, primíparas e multíparas no rebanho, aos manejos nutricional e reprodutivo, a problemas sanitários, à distribuição de chuvas e à disponibilidade de forragens ao longo dos anos, no período avaliado.

\section{Agradecimento}

À Empresa Estadual de Pesquisa Agropecuária do Estado da Paraíba, pela cessão dos dados.

\section{Literatura Citada}

ANDERSON, L.L. Cerdos. In: HAFEZ, E.S.S.E. (Ed.) Reprodución e inseminacion artificial em animales. México: Interamericana, 1986. p.341-368.

ARAÚJO FILHO, J.A.; SILVA, N.L. Alternativas para o aumento da produção de forragem na caatinga. In: SIMPÓSIO NORDESTINO DE ALIMENTAÇÃO DE RUMINANTES, 5., 1994, Salvador. Anais... Salvador: Sociedade Nordestina de Produção Animal, 1994. p.121-133.

ARAÚJO FILHO, J.A.; LEITE, E.R.; MESQUITA, R.C.M. Dieta e desempenho de caprinos em bancos de proteína na região de Sobral. Ceará: EMBRAPA/CNPC, 1990. 14p. (Boletim de Pesquisa, 15)

ARGHEORE, E.M.; CHIBANGA, C.O.C.; LUNGU, J.C.N. Effect of plane of nutrition on body weight and birth weight of pregnant Gwembe Valley goats in Zambia. Small Ruminant Research, v.9, p.201-208, 1992.

AWEMU, E.M.; NWAKALOR, L.N.; ABUBAKAN, B.Y. Technical Note: Enviromental influences on preweaning mortality and reproductive performance of Red Sokoto does. Small Ruminant Research, v.34, p.161-165, 1999.

CHAWLA, D.S.; BHATNAGAR, D.S.; MISHRA, R.R. Factors affecting kid mortality in dairy goats. Indian Journal of Animal Science, v.52, n.3, p.166-171, 1982.

ECONOMIDES, S.; LOUCA, A. Flock management in intensive goat systems. In: INTERNATIONAL CONFERENCE ON GOATS, 4., 1987, Brasília. Proceedings... Brasília: EMBRAPA/CNPC, 1987. v.1, p.867-883.

FEHR, P.M. Growth. In: Goat production. New York: Academic Press, 1981. p.253-283.

FIGUEIREDO, E.A.P.; PANT, K.P. Evaluation of goat breeds in the tropical northeast Brazil. II. An analysis of age at death of kids. Pesquisa Agropecuária Brasileira, v.17, n. 5, p. 803-808, 1982.

GONÇALVES, H.C. Fatores genéticos e de meio em algumas características produtivas e reprodutivas de caprinos. Viçosa, MG: Universidade Federal de Viçosa, 1996. 141p. Tese (Doutorado em Melhoramento Genético Animal) Universidade Federal de Viçosa, 1996.

GUIMARÃES FILHO, C. Eficiência reprodutiva de caprinos no Nordeste semi-árido: limitações e possibilidades. (S.I.):

R. Bras. Zootec., v.33, n.6, p.1711-1720, 2004 (Supl. 1) 
Petrolina. EMBRAPA-Centro de Pesquisa Agropecuária do Trópico Semi-Árido, 1983. 40p. (Documentos, 20).

JAGTAP, D.Z.; BANSOD, R.S.; BELHE, N.D. Nongenetic factors influencing reproductive traits in Angora crossbred goats. Indian Journal of Animal Science, v.61, n.11, p.1236-1237, 1991.

MAJID, A.M.; CARTWRIGHT, T.C.; YAZMAN, J.A. et al. Performance of five breeds of dairy goats in southern United States. 1. Reproductives traits and maturing pattern. World Review of Animal Production, v.57, n.2, p.15-23, 1993.

MORAND-FEHR, P.; SAUVANT, D. Alimentación de cabras. In: CHURCH, D.C. (Ed.) Alimentos y alimentación del ganado. Montevideo: Hemisferio Sur, Tomo 2., 1984. p.552-577.

OLIVEIRA, E.R. Suplementação protéica e energética para cabras leiteiras. In: SIMPÓSIO NORDESTINO SOBRE CAPRINOS E OVINOS DESLANADOS, 1992, Taperoá. Anais... Taperoá: Associação Paraibana dos Criadores de Caprinos e Ovinos, 1992. p.1-16.

PETER, A.M.B. Composição botânica e química da dieta de bovinos, caprinos e ovinos em pastoreio associativo na caatinga nativa do semi-árido de Pernambuco. Recife: Universidade Federal Rural de Pernambuco, 1992. 86p. Dissertação (Mestrado em Zootecnia) - Universidade Federal Rural de Pernambuco, 1992.

PU, J.; LIU, X.; PELANT, R. Crossbreeding for milk production in Sichuan province, China. In: INTERNATIONAL CONFERENCE ON GOATS, 4., 1987, Brasília. Proceedings... Brasília: EMBRAPA/CNPC, 1987.v.1.p.1445.

QUITTET, E. La cabra. Guia pratica para el ganadero. Madrid: Mundi-Prensa, 1978. 32p.

RAMÍREZ, R.G.; LOYO, A.; MORA, R. et al. Forage intake and nutrition in Northeastern Mexico. Journal of Animal Science, v.69, p.879-885, 1991.

RUVUNA, F.; CARTWRIGHT, T.C.; TAYLOR, J.F. et al. Factors affecting body weight of East African and Galla goats. Small Ruminant Research, v.4, n.4, p.339-347, 1991.

SAMPAIO, J.M.C.; CAFEZEIRO, P.T.M.; ASSIS, J.V. et al. Criação de cabras leiteiras. Brasília: EMBRATER, 1984.
SCHACHT, W.H.; MALECHEK, J.C. Nutrition of goats as influenced by thinning and clearing of deciduos wooland in Northeastern Brazil. Journal of Animal Science, v.67, p.2487-2497, 1989.

SHELTON, M. Reproduction and breeding of goats. Journal of Dairy Science, v.61, p.994-1010, 1978.

SILVA, A.E.D.F.; UNANIAN, M.M.; BARROS, N.N. Efeito da suplementação no desempenho produtivo e reprodutivo de ovelhas deslanadas no Nordeste. Pesquisa Agropecuária Brasileira, v.21, n.9, p.987-997, 1986.

SILVA, A.G.S.; SILVA, H.M. Suplementação alimentar durante a estação seca de desempenho de caprinos nativos no semiárido do Nordeste brasileiro. Arquivo Brasileiro de Medicina Veterinária e Zootecnia, v.39, n.4, p.611-622, 1987.

SILVA, F.L.R.; ARAÚJO, A.M. Desempenho produtivo em caprinos mestiços no semi-árido do Nordeste do Brasil. Revista Brasileira de Zootecnia, v.29, n.4, p.1028-1035, 2000.

SILVEIRA, V.C.P.; LÓPEZ, J.; RODRIGUES, F.E. Influência da nutrição materna e do sexo na reserva energética do cordeiro ao nascer. Revista da Sociedade Brasileira de Zootecnia, v.21, n.2, p.243-249, 1992.

SOLANKI,G.S. Feeding habits and grazing behaviour of goats in semi-arid region of India. Small Ruminant Research, v.14, p.39-43, 1994.

STEINE, T.A. Factors affecting traits of economic importance in goat. Meld. Nor. Landbrukshogsk, v.54, p.1-30, 1975.

UNIVERSIDADE FEDERAL DE VIÇOSA - UFV. SAEG Sistema de Análises Estatísticas e Genéticas. Viçosa, MG: 1992. 68p. (Manual do usuário).

YAPPI, C.V.; BOYLAN, W.J.; ROBINSON, R. Factors associated with causes of preweaning lamb mortality. Preventive Veterinary Medicine, v.10, n.1-2, p.145-152, 1990. 